ACta Univ. Sapientiae, Informatica 11, 1 (2019) 52-64

DOI: $10.2478 /$ ausi-2019-0004

\title{
New results on connected dominating structures in graphs
}

\author{
Libin Chacko SAMUEL \\ CHRIST (Deemed to be University) \\ email: \\ Mayamma JOSEPH \\ CHRIST (Deemed to be University) \\ email: \\ libin.samuel@res.christuniversity.in mayamma.joseph@christuniversity.in
}

\begin{abstract}
A set of vertices in a graph is a dominating set if every vertex not in the set is adjacent to at least one vertex in the set. A dominating structure is a subgraph induced by the dominating set. Connected domination is a type of domination where the dominating structure is connected. Clique domination is a type of domination in graphs where the dominating structure is a complete subgraph. The clique domination number of a graph $\mathrm{G}$ denoted by $\gamma_{k}(\mathrm{G})$ is the minimum cardinality among all the clique dominating sets of G. We present few properties of graphs admitting dominating cliques along with bounds on clique domination number in terms of order and size of the graph. A necessary and sufficient condition for the existence of dominating clique in strong product of graphs is presented. A forbidden subgraph condition necessary to imply the existence of a connected dominating set of size four also is found.
\end{abstract}

\section{Introduction}

The study of domination in graphs is to a great extent a result of the study of games and recreational mathematics. It began when C.F. De Jaenisch attempted to determine the minimum number of queens that can be placed on

Computing Classification System 1998: G.2.2

Mathematics Subject Classification 2010: 05C69

Key words and phrases: dominating structure, clique domination, connected domination, strong product, tensor product 
an $n \times n$ chess board so that all squares are either attacked by a queen or are occupied by a queen [10]. Domination in graph can be defined in a similar terms as finding a set of vertices in a graph such that every vertex in the graph is either adjacent to some vertex in the set or is in the set. Further development in domination was observed in late 1950s with Claude Berge [3] introducing coefficient of external stability which is now known as domination number. A set of vertices in a graph is a dominating set if every vertex in the graph which is not in the dominating set is adjacent to one or more vertices in the dominating set. The domination number, $\gamma(\mathrm{G})$, of a graph $\mathrm{G}$ is the minimum number of vertices in a dominating set. Over the course of time different types of domination in graphs such as total domination, connected domination and independent domination were developed by imposing conditions on the dominating set. For example a connected dominating set is a dominating set that induces a connected subgraph. [17, 9, 7, 12, 2, 16].

A dominating structure in a graph is a subgraph induced by its dominating set. Identification of graphs possessing specific types of dominating structures is a problem that caught the attention of several researchers. In this paper we are exploring graphs having complete graphs as a dominating structure. Every graph referred to in this article is finite, undirected, simple and connected. $[5,14,4]$ A clique dominating set is a dominating set that induces a complete subgraph. A clique dominated graph is a graph that contains a clique as a dominating structure. Cozzens and Kelleher were the first to deal with dominating cliques. The clique domination number, $\gamma_{k}(\mathrm{G})$, of a graph $\mathrm{G}$ is the minimum number of vertices in a clique dominating set.

The concept of domination is very useful to model several real-world problems such as social networks, bus routing, land surveying, computer and communication networks . Facility allocation is another area wherein one finds one of the most important applications of domination; in particular connected domination and clique domination. It involves optimal placement of facilities in a given area. $[6,7,8]$ For example let us consider the problem of effective allocation of airports and air routes of a country. The airports in important cities of a country are connected with each other, while every other airport is connected with that of at least one of the important cities. Another instance is a wireless sensor network which is comprised of autonomous sensor nodes where the connected dominating set enable faster communication by forming a virtual network backbone for information and control routing.[15, 11, 13, 1] 


\section{Related results}

It is note worthy that every graph need not have a dominating clique. The smallest clique being $\mathrm{K}_{1}$, the smallest dominating clique is a single vertex. It is clear that a graph with a dominating vertex has a star as spanning tree. Wolk [18] gave the necessary condition for the graphs to have dominating clique of size one and he called such a dominating clique a central vertex or a central point. Dominating clique of size two is an edge called dominating edge.

Theorem 1 (Wolk [18]) If $G$ is a finite connected graph with no induced $\mathrm{P}_{4}$ or $\mathrm{C}_{4}$, then $G$ has a dominating vertex.

Cozzens and Kelleher [5] extended the theorem to get a forbidden subgraph condition to establish the existence of a dominating clique, which is presented below

Theorem 2 (Cozzens and Kelleher [5]) If $G$ is a connected graph that has no induced $P_{5}$ or $C_{5}$ then $G$ has a dominating clique.

Although the above result ensures the existence of a dominating clique, it does not specify the size of the dominating clique. In the direction,Cozzens and Kelleher [5] have explored the problem of identifying graphs possessing connected dominating set of size 3 .

The notation $K_{n+p}[5]$ represents the complete graph $K_{n}$ on $n$ vertices along with $n$ pendants, one at each vertex of the complete graph. For example $K_{3+p}$ is the net graph. $K_{3+p}$ and $K_{4+p}$ are shown in the Figure 1.
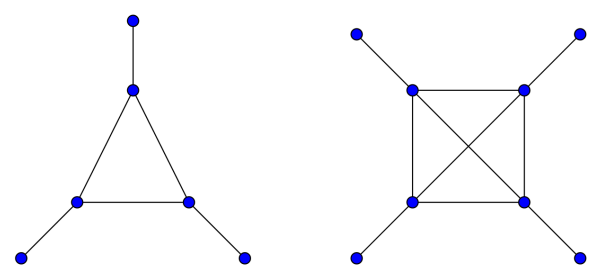

Figure 1: The graphs $\mathrm{K}_{3+p}$ and $\mathrm{K}_{4+\mathrm{p}}$

Note that connected dominating sets of size one and two respectively are defined uniquely whereas a connected dominating set of size three is either a $\mathrm{P}_{3}$ or a $\mathrm{K}_{3}$. 

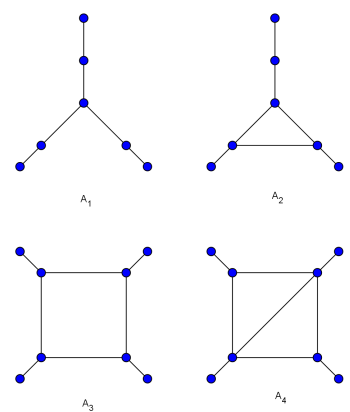

Figure 2: The graphs $A_{1}, A_{2}, A_{3}$ and $A_{4}$

The characterization obtained by Cozzens and Kelleher [5] was in terms of a class $\mathcal{A}=\left\{\mathrm{P}_{6}, \mathrm{C}_{6}, \mathrm{~K}_{4+\mathrm{p}}, \mathrm{A}_{1}, \mathrm{~A}_{2}, \mathrm{~A}_{3}, \mathrm{~A}_{4}\right\}$ of graphs where the graphs $A_{1}, A_{2}$, $A_{3}$ and $A_{4}$ are as shown Figure 2 .

Theorem 3 (Cozzens and Kelleher [5]) If G is a finite, connected graph with three or more vertices that has none of the graphs in Class $\mathcal{A}$ as an induced subgraph, then $\mathrm{G}$ has a connected dominating set of size three.

We have settled the problem of obtaining a necessary condition for graphs to have a connected dominating set of size of 4 and the result is presented section 5. First we will explore the bounds for clique domination number $\gamma_{k}$.

\section{Bounds for clique domination number}

Recall that a private neighbour of a vertex $v$ with respect to the set $\mathrm{K}$ is a vertex adjacent to only $v$ from the set K. First we present the following proposition.

Proposition 4 If $\mathrm{K}$ is a minimal dominating clique of a graph $\mathrm{G}$, then every vertex in $\mathrm{K}$ has a private neighbour.

Proof. On the contrary, assume that there is a vertex $v \in \mathrm{K}$ having no private neighbor. Then $v$ is adjacent to every vertex in $\mathrm{K}$ and $v$ will have no private neighbour. This implies that $\mathrm{K}-\{v\}$ is a smaller dominating clique contained in $\mathrm{K}$, which contradicts the minimality of $\mathrm{K}$.

The bound obtained by Ore [10] for domination number, is true for clique domination as given below. 
Proposition 5 If a connected graph $\mathrm{G}$ of order $\mathrm{n}$ has a dominating clique, then $\gamma_{k}(\mathrm{G}) \leq \mathrm{n} / 2$.

Proof. Assume that $\gamma_{k}(G)>n / 2$. Then $\gamma_{k}$-set of $G$ being a minimal dominating clique, it is clear that there exists a vertex $v \in \gamma_{k}$-set of $G$ which does not have a private neighbor which is a contradiction to the proposition 3.1.

Remark 6 The bound obtained in Proposition 3.2 is sharp and $\mathrm{K}_{\mathrm{n}+\mathrm{p}}$ is a class of graph that attains the bound. There are $2 \mathrm{n}$ vertices in $\mathrm{K}_{\mathrm{n}+\mathrm{p}}$ and the minimum dominating set is of size $\mathrm{n}$.

It is obvious that the domination number serves as a lower bound for clique domination number. Then the following inequality follows immediately.

Proposition 7 If the graph $\mathrm{G}$ has a dominating clique, then $\gamma(\mathrm{G}) \leq \gamma_{\mathrm{k}}(\mathrm{G})$ $\leq \omega(\mathrm{G})$ where $\omega(\mathrm{G})$ is the clique number of the graph.

Remark 8 Let $\mathrm{G}$ and $\mathrm{H}$ be two graphs. The corona product $\mathrm{G} \circ \mathrm{H}$, is the graph obtained by taking one copy of $\mathrm{G}$ and $|\mathrm{V}(\mathrm{G})|$ copies of $\mathrm{H}$ and by joining each vertex of the $i$-th copy of $\mathrm{H}$ to the $i$-th vertex of $\mathrm{G}$, where $1 \leq i \leq|\mathrm{V}(\mathrm{G})|$. The graph $\mathrm{K}_{\mathrm{r}} \circ \mathrm{K}_{\mathrm{s}}$, where $\mathrm{r} \geq \mathrm{s}$ has $\gamma\left(\mathrm{K}_{\mathrm{r}} \circ \mathrm{K}_{\mathrm{s}}\right)=\gamma_{\mathrm{k}}\left(\mathrm{K}_{\mathrm{r}} \circ \mathrm{K}_{\mathrm{s}}\right)=\omega\left(\mathrm{K}_{\mathrm{r}} \circ \mathrm{K}_{\mathrm{s}}\right)=\mathrm{r}$.

The next theorem gives a bound for the clique domination number of a graph in terms of its size.

Theorem 9 If $G$ is a graph with $\mathrm{m}$ edges possessing a dominating clique, then

$$
\gamma_{k}(G) \leq \frac{\sqrt{1+8 m}-1}{2}
$$

Proof. We know that a clique of size $\gamma_{k}$ has $\frac{\gamma_{k}\left(\gamma_{k}-1\right)}{2}$ edges. Therefore, $m \geq$ $\frac{\gamma_{k}\left(\gamma_{k}-1\right)}{2}+\gamma_{k}$ so that $m \geq \frac{\gamma_{k}\left(\gamma_{k}+1\right)}{2}$. By solving which we will get $\gamma_{k}(G)$ $\leq \frac{\sqrt{1+8 m}-1}{2}$ or $\gamma_{k}(G) \leq \frac{-\sqrt{1+8 m}-1}{2}$. Latter being impossible can be

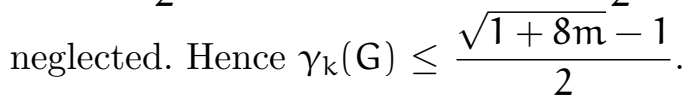

Remark 10 We can observe that the bound is sharp and $\mathrm{K}_{\mathrm{n}+\mathrm{p}}$ is a class of graph that attains the bound. Figure 1 shows the graphs $\mathrm{K}_{3+\mathrm{p}}$ and $\mathrm{K}_{4+\mathrm{p}}$. 
Obviously a graph $\mathrm{G}$ with maximum degree $\Delta=\mathrm{n}-1$ has a dominating vertex as the vertex with degree $n-1$ itself is a dominating vertex. We now consider graphs with $\Delta=n-2$ and obtain the following theorem.

Theorem 11 If $\mathrm{G}$ is a connected graph with maximum degree $\Delta=\mathfrak{n}-2$, then $\mathrm{G}$ has a dominating edge.

Proof. Since $\Delta=\mathrm{n}-2$, there exists a vertex, say $v$ in $\mathrm{G}$ which is adjacent to all but one vertex (obviously excluding $v$ ), say $w$, of the graph. But $\mathrm{G}$ is a connected graph and $w$ is not adjacent to $v$ which implies that $w$ is adjacent to a neighbor of $v$ say $u$, so that $u v$ is a dominating edge of $G$.

\section{Clique domination in product of graphs}

Clique domination problem for two types of graph product namely Lexicographical product and Cartesian product has been studied[4]. We now extend for clique domination in tensor products strong products of graphs.

The tensor product $\mathrm{G} \times \mathrm{H}$ of graphs $\mathrm{G}$ and $\mathrm{H}$ is a graph such that the vertex set of $\mathrm{G} \times \mathrm{H}$ is the Cartesian product $\mathrm{V}(\mathrm{G}) \times \mathrm{V}(\mathrm{H})$; and any two vertices $(u, v)$ and $\left(u^{\prime}, v^{\prime}\right)$ are adjacent in $\mathrm{G} \times \mathrm{H}$ if and only if $u$ is adjacent with $u^{\prime}$ in $G$ and $v$ is adjacent with $v^{\prime}$ in $\mathrm{H} .[17,9]$

We can understand by the definition of tensor product of graphs that any vertex $\left(u^{\prime}, v^{\prime}\right)$ is not adjacent to any other vertex $\left(u^{\prime}, v_{i}\right)$ and $\left(u_{j}, v^{\prime}\right), \forall v_{i} \in$ $\mathrm{V}(\mathrm{H})$ and $\forall \mathrm{u}_{j} \in \mathrm{V}(\mathrm{G})$. For any two graphs $\mathrm{G}$ and $\mathrm{H}, \gamma(\mathrm{G} \times \mathrm{H}) \geq 2$. For any graph $G$ of order $n, G \times K_{1}$ is $\overline{K_{n}}$. And $G \times K_{2}$ is a bipartite graph.

Proposition 12 For complete graphs $\mathrm{K}_{\mathrm{r}}$ and $\mathrm{K}_{\mathrm{s}}, \gamma_{\mathrm{k}}\left(\mathrm{K}_{\mathrm{r}} \times \mathrm{K}_{\mathrm{s}}\right)=3$, if $r, s \geq 3$

Proof. We can observe that the tensor product of two complete graphs $K_{n}$ and $\mathrm{K}_{\mathrm{m}}$ is a graph with any vertex $\left(\boldsymbol{u}_{i}, v_{j}\right)$ is adjacent to all vertices $\left(u_{k}, v_{l}\right)$, $\forall \mathrm{k} \neq \mathrm{i}$ and $\forall \mathrm{l} \neq \mathrm{j}$ Therefore, by choosing three vertices $\left(u_{i_{1}}, v_{j_{1}}\right),\left(u_{i_{2}}, v_{j_{2}}\right)$ and $\left(u_{i_{3}}, v_{j_{3}}\right)$ where $i_{1} \neq i_{2} \neq i_{3}$ and $j_{1} \neq j_{2} \neq j_{3}$ we obtain a dominating clique, thus proving that $\gamma_{k}\left(K_{r} \times K_{s}\right) \leq 3$. As we have observed earlier, we require at least two vertices to dominate a graph. And if we consider an edge, the two vertices in the edge say $\left(u_{i_{1}}, v_{j_{1}}\right)$ and $\left(u_{i_{2}}, v_{j_{2}}\right)$ can dominate all the vertices but $\left(u_{i_{1}}, v_{j_{2}}\right)$ and $\left(u_{i_{2}}, v_{j_{1}}\right)$, hence the graph cannot be dominated by an edge. Therefore $\mathrm{K}_{3}$ is the smallest clique dominating the tensor product $\mathrm{K}_{\mathrm{r}} \times \mathrm{K}_{\mathrm{s}}$

The strong product $\mathrm{G} \otimes \mathrm{H}$ of two graphs $\mathrm{G}$ and $\mathrm{H}$ is the graph with $\mathrm{V}(\mathrm{G} \otimes \mathrm{H})=$ $V(G) \times V(H)$ and $\left(u, u^{\prime}\right)\left(v, v^{\prime}\right) \in E(G \otimes H)$ if and only if either $u v \in E(G)$ 
and $u^{\prime}=v^{\prime}$ or $u=v$ and $u^{\prime} v^{\prime} \in E(H)$ or $u v \in E(G)$ and $u^{\prime} v^{\prime} \in E(H)$. Note that if $\mathrm{C} \subseteq \mathrm{V}(\mathrm{G} \otimes \mathrm{H})$, then the G-projection and H-projection of $\mathrm{C}$ are, respectively, the sets $\mathrm{C}_{\mathrm{G}}=\{\mathrm{u} \in \mathrm{V}(\mathrm{G}):(\mathrm{u}, \mathrm{b}) \in \mathrm{C}$ for some $\mathrm{b} \in \mathrm{V}(\mathrm{H})\}$ and $\mathrm{C}_{\mathrm{H}}=\{v \in \mathrm{V}(\mathrm{H}):(\mathrm{a}, v) \in \mathrm{C}$ for some $\mathrm{a} \in \mathrm{V}(\mathrm{G})\} .[17,9]$

Theorem 13 The graph $\mathrm{G} \otimes \mathrm{H}$ has a dominating clique if and only if the graphs $\mathrm{G}$ and $\mathrm{H}$ have dominating cliques.

Proof. Suppose $G \otimes H$ has a dominating clique. Let $C \subseteq V(G \otimes H)$ be the dominating clique of $\mathrm{G} \otimes \mathrm{H}$. Consider the projections $\mathrm{C}_{\mathrm{G}}$ and $\mathrm{C}_{\mathrm{H}}$ of $\mathrm{C}$ on $\mathrm{G}$ and $H$ respectively. We claim that $C_{G}$ is a dominating clique of $G$ and $C_{H}$ is a dominating clique of $\mathrm{H}$. Strong product being commutative, it is sufficient to show that $C_{G}$ is a dominating clique of $G$. Let $u, u^{\prime} \in C_{G}$ be distinct vertices. By the definition of projection we can observe that there exist adjacent vertices $(u, v)$ and $\left(u^{\prime}, v^{\prime}\right)$ in $C$. We know that $(u, v)$ and $\left(u^{\prime}, v^{\prime}\right)$ are adjacent in $G \otimes H$ implies that either $u u^{\prime} \in E(G)$ and $v=v^{\prime}$ or $u=u^{\prime}$ and $v v^{\prime} \in E(H)$ or $u u^{\prime} \in E(G)$ and $v v^{\prime} \in E(H)$. Since $u$ and $u^{\prime}$ are distinct we can easily conclude that $u u^{\prime} \in E(G)$. Therefore $C_{G}$ forms a clique in $G$. Now, to show that $C_{G}$ is a dominating set. Let $u_{1} \notin C_{G}$ be vertex of $G$. There exists a vertex $\left(u_{1}, v_{1}\right)$ in $\mathrm{G} \otimes \mathrm{H}$. Since $\mathrm{C}$ is a dominating clique in $\mathrm{G} \otimes \mathrm{H}$, there exists a vertex $\left(\mathrm{u}_{0}, v_{0}\right) \in$ $C$ adjacent to $\left(u_{1}, v_{1}\right)$. Since $u_{0}$ and $u_{1}$ are distinct, by definition of an edge in strong product $u_{1}$ and $u_{0}$ are adjacent. Therefore, $C_{G}$ is a dominating clique of $\mathrm{G}$.

Conversely, let $S_{G}$ and $S_{H}$ be the dominating cliques in the graphs $G$ and $H$. We claim that $S_{G} \times S_{H}$ forms a dominating clique in $G \otimes H$. Firstly to show that $S_{G} \times S_{H}$ is a clique in $G \otimes H$. Let $(u, v)$ and $\left(u^{\prime}, v^{\prime}\right)$ be two distinct vertices in $S_{G} \times S_{H}$. Either $u=u^{\prime}$ or $u u^{\prime} \in E(G)$ and $v=v^{\prime}$ or $v v^{\prime} \in E(H)$. Either ways $(u, v)$ and $\left(u^{\prime}, v^{\prime}\right)$ are adjacent. Hence, $S_{G} \times S_{H}$ is a clique in $G \otimes H$. Now to show that $S_{G} \times S_{H}$ dominates $G \otimes H$. Consider a vertex $\left(u_{1}, v_{1}\right)$ not in $S_{G} \times S_{H}$. If $u_{1}$ not in $S_{G}$ then there exists a $u_{0}$ in $S_{G}$ adjacent to $u_{1}$ in $G$ and a $v_{0}$ in $S_{\mathrm{H}}$ where $v_{0}=v_{1}$ or $v_{0}$ and $v_{1}$ are adjacent in $\mathrm{H}$. By the definition of strong product of graphs $\left(u_{1}, v_{1}\right)$ is adjacent to $\left(u_{0}, v_{0}\right)$. And if $u_{1}$ is in $S_{G}$ since $\left(u_{1}, v_{1}\right)$ not in $S_{G} \times S_{H}$ there exist $v_{0} \neq v_{1}$ in $S_{H}$ dominating $v_{1}$ in $H$. Owing to the definition of strong product of graphs $\left(u_{1}, v_{1}\right)$ is adjacent to $\left(u_{1}, v_{0}\right)$. Therefore, $S_{G} \times S_{H}$ forms a dominating clique in $G \otimes H$.

Theorem 14 If $\mathrm{G}$ and $\mathrm{H}$ are connected graphs with dominating cliques, then $\gamma_{k}(G \otimes H)=\gamma_{k}(G) \times \gamma_{k}(H)$ 
Proof. Let $S_{G}$ and $S_{H}$ be the $\gamma_{k}$ sets of $G$ and $H$ respectively. We know that $\mathrm{S}_{\mathrm{G}} \times \mathrm{S}_{\mathrm{H}}$ forms a dominating clique in $\mathrm{G} \otimes \mathrm{H}$. This implies

$$
\gamma_{k}(G \otimes H) \leq \gamma_{k}(G) \times \gamma_{k}(H)
$$

To show that $\gamma_{k}(G \otimes H) \geq \gamma_{k}(G) \times \gamma_{k}(H)$ we need to show that $\forall\left(u_{i}, v_{i}\right) \in$ $S_{G} \times S_{H},\left(u_{i}, v_{i}\right) \in \gamma_{k}$-set of $(G \otimes H) . u_{i} \in S_{G}$ implies $u_{i}$ has a private neighbor say $u_{1}$. Similarly $v_{i}$ has a private neighbor $v_{1}$. We claim that $\left(u_{1}, v_{1}\right)$ is a private neighbor of $\left(u_{i}, v_{i}\right)$, i.e. there is no $\left(u_{2}, v_{2}\right)$ adjacent to $\left(u_{1}, v_{1}\right)$ in $S_{G} \times S_{H}$. If there exists a vertex, say, $\left(u_{2}, v_{2}\right)$ adjacent to $\left(u_{1}, v_{1}\right)$ then by definition of strong product $u_{2}=u_{1}$ and $v_{1} v_{2} \in E(H)$ or $u_{1} u_{2} \in E(G)$ and $v_{2}=v_{1}$ or $u_{1} u_{2} \in E(G)$ and $v_{1} v_{2} \in E(H)$ all contradicting the fact that $u_{1}$ is the private neighbor of $u_{i}$ and $v_{1}$ is the private neighbor of $v_{i}$. Which implies that $\forall$ $\left(u_{i}, v_{i}\right) \in S_{G} \times S_{H},\left(u_{i}, v_{i}\right) \in \gamma_{k}$-set of $(G \otimes H)$. Hence $S_{G} \times S_{H}$ is a minimal dominating clique of $G \otimes H$. To show that $S_{G} \times S_{H}$ is a $\gamma_{k}$ set of $G \otimes H$, assume the contrary, if $S_{G} \times S_{H}$ is not a $\gamma_{k}$ set of $G \otimes H$, then there exist a smaller dominating clique $T$ whose projections $T_{G}$ and $T_{H}$ forms a smaller dominating clique for $\mathrm{G}$ and $\mathrm{H}$ respectively hence contradicting the minimality of $\mathrm{S}_{\mathrm{G}}$ and $\mathrm{S}_{\mathrm{H}}$.

\section{Graphs with connected dominating structure of order four}

A forbidden subgraph condition necessary for a graph to have a connected dominating set of size three was found by Cozzens and Kelleher [5]. We discuss a forbidden subgraph condition necessary to have a connected dominating set of size four. There are 6 connected graphs on four vertices :- $\mathrm{K}_{4}, \mathrm{C}_{4}, \mathrm{P}_{4}$, Claw $\left(\mathrm{K}_{1,3}\right)$, Paw and Diamond $\left(\mathrm{K}_{4}-\mathrm{e}\right)$. Therefore a connected dominating set of size four can be any of the above mentioned graph.

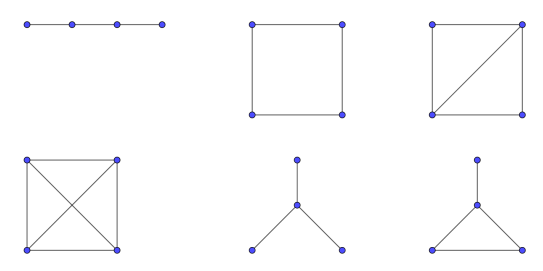

Figure 3: Connected graphs of order four 
Theorem 15 If $G$ is a finite, connected graph with four or more vertices that has none of the graphs in $\mathcal{B}$ (Fig. 4) as an induced subgraph, then $G$ has a connected dominating structure of order four.
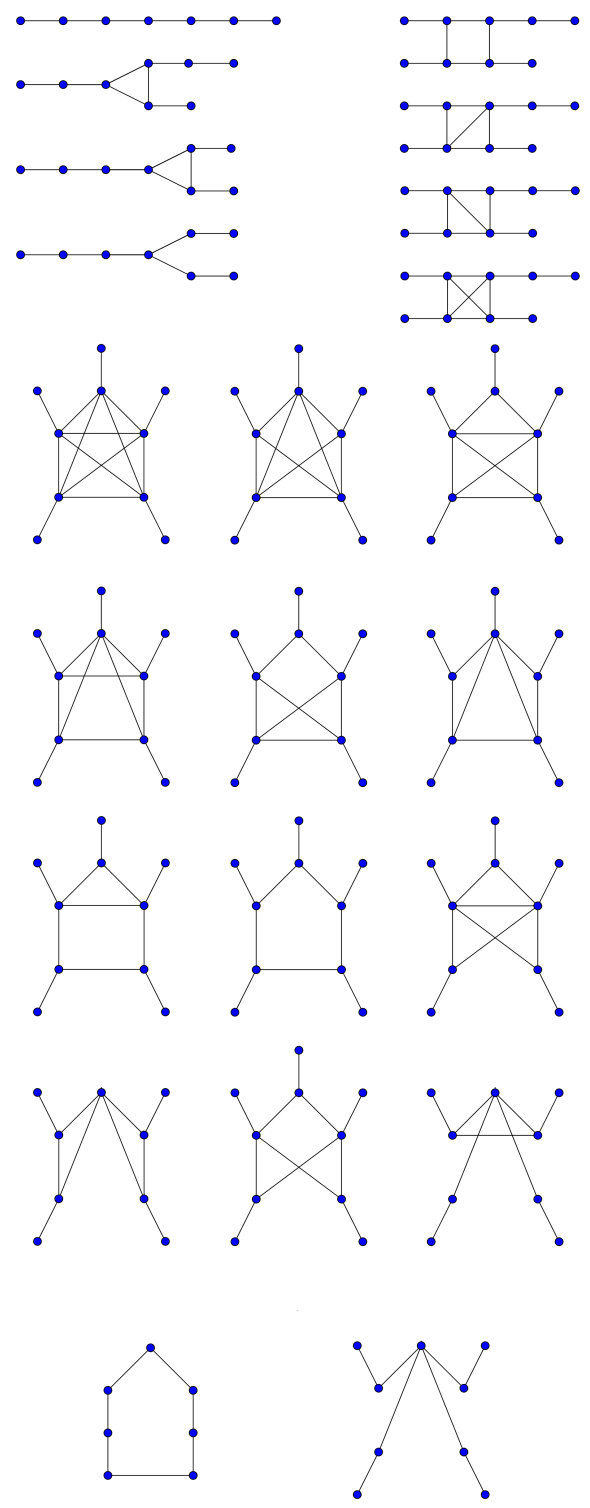

Figure 4: Class $\mathcal{B}$ 
Proof. By induction on $n$, the order of graph $\mathrm{G}$,

(i) The theorem is true when $n=4$.

(ii) Assume that any finite connected graph with $n$ vertices, $n \geq 4$, that has none of the graphs in $\mathcal{B}$ as an induced subgraph has a connected dominating structure of size four.

(iii) Let $G$ be a finite connected graph on $n+1$ vertices, where $n \geq 4$, that has none of the graphs in class $\mathcal{B}$ as an induced subgraph. Let $v$ be vertex of $G$ which is not a cut vertex. Consider the graph $G^{\prime}$, subgraph of $G$ induced by all vertices of $G$ excluding $v$. Since $G^{\prime}$ is a finite connected graph with $n$ vertices having no graphs from class $\mathcal{B}$ as an induced subgraph, by the induction hypothesis $G^{\prime}$ has a connected dominating structure of order four.

Let $S=\{a, b, c, d\}$ induce the connected dominating structure of order four of $G^{\prime}$. If $v$ is adjacent to any vertex in $S$, then $S$ dominates $G$ also.

Suppose that in $G, v$ is not adjacent to any vertex in $S$. Since $G$ is connected, $v$ must be adjacent to some vertex of $G$, say $x$. And $S$ being the connected dominating set of $\mathrm{G}^{\prime}, x$ must be adjacent to some vertex in $S$. The set $\{a, b, c, d, x\}$ induces a connected graph of 5 vertices. Therefore, the graph induced by $S \cup N(S) \cup\{v\}$ has one of the graphs from $\mathcal{B}$ as a subgraph, not necessarily induced, i.e, there might be edges between the pendant vertices and other vertices. If there are no edges between the pendant vertices and the other vertices, this implies that the subgraphs are induced, which contradicts the assumption that $G$ has none of the graphs in class $\mathcal{B}$ as an induced subgraph.

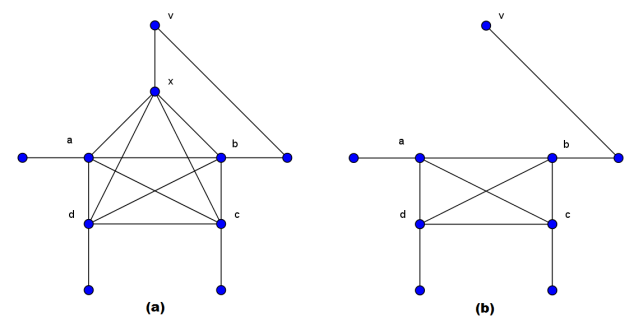

Figure 5: Graphs used in the proof of Theorem 15

Suppose $G$ has at least one edge between the pendant vertices. If $G$ has exactly one edge between vertices as shown in Figure 5(a), then $G$ has an induced subgraph shown in Figure 5(b), which is a forbidden subgraph from class $\mathcal{B}$. 


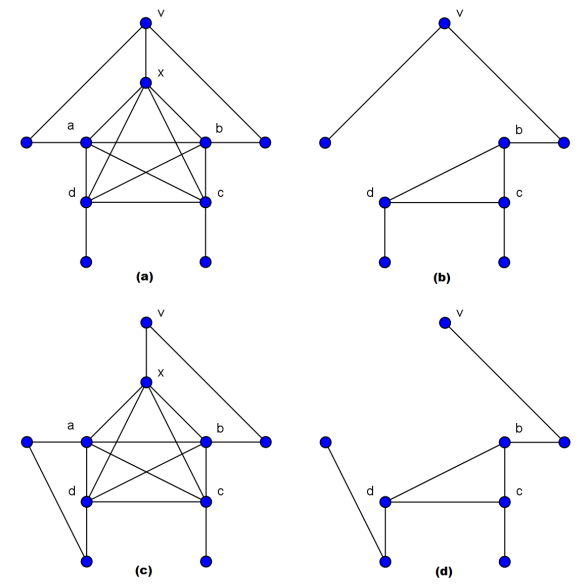

Figure 6: Graphs used in the proof of Theorem 15

If $G$ has exactly two edges between the pendant vertices as shown in Figure 6(a) or 6(c), then G has an induced subgraph shown in Figure 6(b) or 6(d), which is a forbidden subgraph from class $\mathcal{B}$.

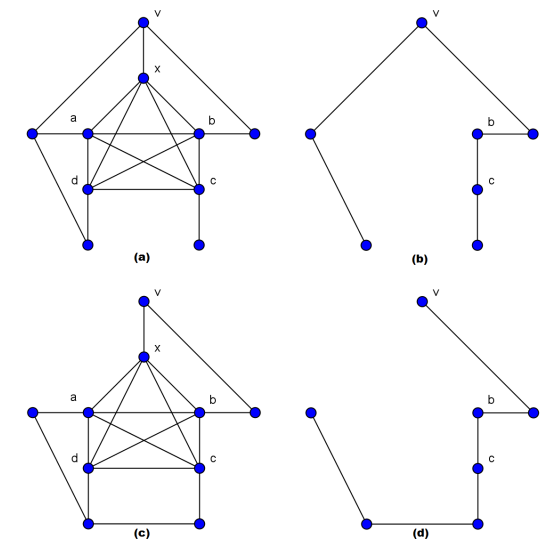

Figure 7: Graphs used in the proof of Theorem 15

If $G$ has exactly three edges between the pendant vertices as shown in Figure $7(\mathrm{a})$ or $7(\mathrm{c})$, then $\mathrm{G}$ has an induced $\mathrm{P}_{7}$, which is a forbidden subgraph from class $\mathcal{B}$. 


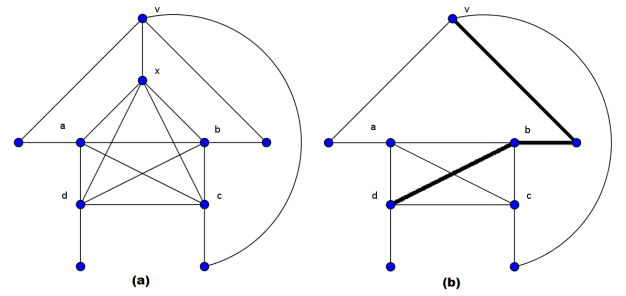

Figure 8: Graphs used in the proof of Theorem 15

If $\mathrm{G}$ has exactly three edges between the pendant vertices as shown in Figure 8(a), then $\mathrm{G}$ has a $\mathrm{P}_{4}$ as shown in Figure 8(b) which is a connected dominating structure of order four.

We can now observe that an edge between the pendant vertices in the graphs in Class $\mathcal{B}$ will lead to obtaining a connected dominating structure of order four or a contradiction to the absence of an induced forbidden structure from class $\mathcal{B}$. Therefore, $\mathrm{G}$ has a connected dominating set of size four.

As we have seen before, the converse of this theorem need not be true. A finite connected graph having graph from $\mathcal{B}$ as an induced subgraph can have a dominating clique of size four. An example is given in Fig. 9.

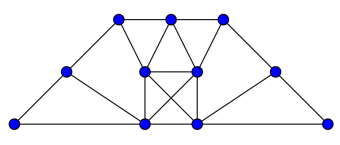

Figure 9: Graph with induced $\mathrm{P}_{7}$ dominated by $\mathrm{K}_{4}$

\section{References}

[1] R. E. Atani, A. H. Karbasi, Application of dominating sets in wireless sensor networks, International Journal of Security and Its Applications 7 (2013) 185202. $\Rightarrow 53$

[2] S. Balamurugan, I. Sahul Hamid, Isolate domination in graphs, Arab Journal of Mathematical Sciences 22 (2016) 232-241. $\Rightarrow 53$

[3] C. Berge, The Theory of Graphs, Dover Publications 2001. $\Rightarrow 53$

[4] S. R. Canoy, T. V. Daniel, Clique domination in a graph, Applied Mathematical Sciences $9116(2015)$ 5749-5755. $\Rightarrow 53,57$ 
[5] M. B. Cozzens, L. L. Kelleher, Dominating cliques in graphs, Discrete Mathematics, 86 (1990) 101-116. $\Rightarrow 53,54,55,59$

[6] M. B. Cozzens, L. L. Kelleher, Dominating sets in social network graphs, Mathematical Social Sciences 16 (1988) 267-279. $\Rightarrow 53$

[7] E. J. Cockayne, R. M. Dawes, S. T. Hedetniemi, Total domination in graphs, Networks, 10 (1980) 211-219. $\Rightarrow 53$

[8] W. Duckworth, B. Mans, Connected domination of regular graphs, Discrete Mathematics 309 (2009) 2305-2322. $\Rightarrow 53$

[9] F. Harary, Graph Theory, Addison-Wesley, Reading, MA 1969. $\Rightarrow 53,57,58$

[10] T. W. Haynes, S. T. Hedetniemi, P. J. Slater, Fundamentals of Domination, MD, 1998. $\Rightarrow 53,55$

[11] M. A. Henning, S. Mukwembi, Domination, radius, and minimum degree, Discrete Mathematics 157 (2009) 2964-2968. $\Rightarrow 53$

[12] I. M. James, History of Topology, UK, 1999. $\Rightarrow 53$

[13] N. J. Kothari, S. K. Vaidya, Some new results on distance k-domination in graphs, International Journal of Combinatorics (2013). $\Rightarrow 53$

[14] D. Kratsch, M. Liedloff, An exact algorithm for the minimum dominating clique problem, Theoretical Computer Science, 385 (2007) 226-240. $\Rightarrow 53$

[15] G. Mekiš, Lower bounds for the domination number and the total domination number of direct product graphs, Discrete Mathematics, 310 (2010) 3310-3317. $\Rightarrow 53$

[16] E. Sampathkumar, H. B. Walikar, The connected domination number of a graph, Jour. Math. Phy. Sci. 13 (1979) 607-613. $\Rightarrow 53$

[17] D. B. West, Introduction to Graph Theory (2nd ed.), Pearson Education, 2002. $\Rightarrow 53,57,58$

[18] E. S. Wolk, A note on "The comparability graph of a tree", Proc. Amer. Math. Sot., 16 (1965) 17-20. $\Rightarrow 54$

Received: March 25, 2019 • Revised: June 24, 2019 